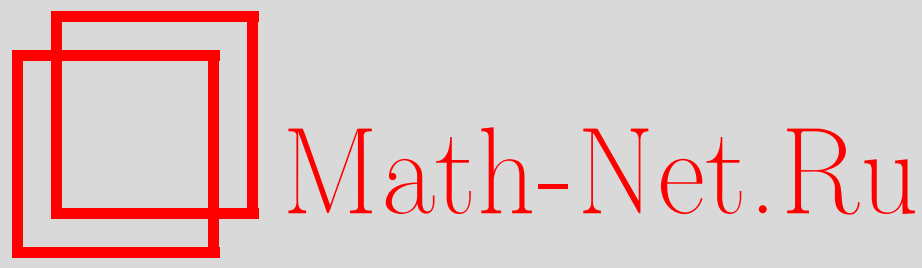

Е. М. Перпер, Нижние оценки временной и объёмной сложности задачи поиска подслова, Дискрет. матем., 2014, том 26, выпуск 2, 58-70

DOI: https://doi.org/10.4213/dm1280

Использование Общероссийского математического портала Math-Net.Ru подразумевает, что вы прочитали и согласны с пользовательским соглашением http://www . mathnet.ru/rus/agreement

Параметры загрузки:

IP : 3.85 .73 .92

26 апреля 2023 г., 12:49:33

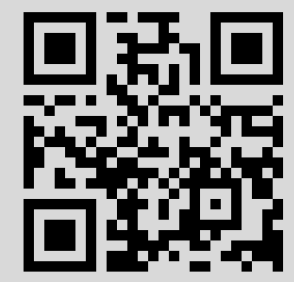




\title{
Нижние оценки временной и объёмной сложности задачи поиска подслова
}

\author{
() 2014 г. Е. М. Перпер*
}

\begin{abstract}
Рассматривается задача поиска подслова в множестве слов. Эта задача состоит в следующем: пусть дано множество слов; требуется для произвольного подслова найти все слова из этого множества, в которых это подслово содержится. В данной работе рассматриваются алгоритмы, позволяющие осуществлять такой поиск, и приведены нижние оценки времени работы этих алгоритмов, а также оценки объёма памяти для самых быстрых алгоритмов.
\end{abstract}

Ключевые слова: подслово, поиск, нижняя оценка.

\section{1. Введение}

Необходимость поиска в большом массиве слов, содержащих данное подслово, часто возникает на практике, в частности, при пользовании словарём. Например, если рассматриваемое слово - имя существительное, находящееся не в именительном падеже, или глагол, находящийся не в инфинитиве, в словаре его может и не оказаться. Если поиск осуществляет не человек, который обычно может легко привести слово к нужной для поиска в словаре форме, а компьютер, это может стать проблемой. Её решением может стать поиск в словаре слов, содержащих некоторое подслово данного слова, например, его основу. Алгоритм, состоящий из перебора всех слов из словаря и проверки каждого из них с целью обнаружения нужного подслова, очевидно, займёт большое время. В данной работе рассматриваются алгоритмы, осуществляющие (в рамках информационно-графовой модели данных, см. $[1,2])$ поиск за время, пропорциональное сумме длины слова-запроса к базе данных и числа слов, содержащих запрос как подслово. Показано, что для некоторых баз данных это время улучшить нельзя. Кроме того, получена нижняя оценка объёма памяти, нужной для таких алгоритмов. Она показывает, что для столь быстрых алгоритмов необходим объём памяти порядка $p n^{2}$, где $p$ - число слов в базе данных, а $n$ - длина каждого слова из базы данных.

\section{2. Основные понятия и формулировка результатов.}

Обозначим $N_{k}=\{1,2, \ldots, k\}$. Пусть $W_{n}^{k}=\cup_{s=1}^{n} N_{k}^{s}\{0\}^{n-s}-$ множество слов длины не более $n$ над алфавитом $N_{k}$. Слово длины $s$ представлено набором длины $n$, первые $s$ элементов которого - из множества $N_{k}$, и если $s<n$, то оставшиеся $n-s$ элементов - нули.

*Место работы: МГУ им. М.В. Ломоносова, e-mail: e_m_perper@mail.ru 
Для каждого слова $w \in W_{n}^{k}$ его длину будем обозначать через $l(w) ; i$-й буквой слова $w \in W_{n}^{k}$, где $i \in\{1, \ldots, n\}$, назовём $i$-й элемент соответствующего слову $w$ набора. В частности, если $i>l(w)$, то $i$-я буква слова $w$ равна 0. Будем обозначать $i$-ю букву слова $w$ через $w[i]$. Через $w[a . . b]$, где $a, b \in \mathbb{N}, 1 \leqslant a \leqslant b \leqslant l(w)$, будем обозначать такое слово $v \in W_{n}^{k}$, что $l(v)=b-a+1$ и $v[i]=w[a+i-1]$ для всех натуральных $i$, не превышающих $l(v)$. Будем называть слово $w[a . . b]$ подсловом слова $w$, начинающимся с его $a$-й буквы и заканчивающимся его $b$-й буквой. Будем говорить, что слова $w \in W_{n}^{k}$ и $v \in W_{n}^{k}$ равны или совпадают (и писать $w=v$ ), если $l(v)=l(w)$ и $v[1]=w[1], v[2]=w[2], \ldots, v[l(v)]=w[l(w)]$ (очевидно, это условие эквивалентно условию $v[1]=w[1], v[2]=w[2], \ldots, v[n]=w[n])$. Для слова $w$ его подслово $w[1 . . q], q \in \mathbb{N}, q \leqslant l(w)$, будем называть началом длины $q$ слова $w$, а подслово $w[l(w)-q+1 . . l(w)], q \in \mathbb{N}, q \leqslant l(w)-$ концом длины $q$ слова $w$. Скажем, что слово $w$ лексикографически следует за словом $v$, а слово $v$ лексикографически предшествует слову $w$, если существует такое натуральное число $i$, не превышающее $n$, что $v[i]<w[i]$ и для всякого натурального числа $j$, меньшего $i, j$-я буква слова $v$ равна $j$-й букве слова $w$. Будем записывать это следующим образом: $w>v$ (либо $v<w)$.

Рассмотрим $X=W_{n}^{k}-$ множество запросов, то есть слов длины не более $n$ над алфавитом $N_{k}$. Рассмотрим также $Y=N_{k}^{n}-$ множество записей. Каждая запись является словом длины $n$ над алфавитом $N_{k}$. Введём бинарное отношение $\rho$, которое позволит устанавливать, когда запись $y \in Y$ удовлетворяет запросу $x \in X$ (отношение поиска):

$$
x \rho y \Leftrightarrow \text { существует } i \in \mathbb{N}: x=y[i . . l(x)+i-1], x \in X, y \in Y \text {. }
$$

Будем рассматривать задачу информационного поиска (ЗИП) $I=<X, V, \rho>$, где $V=\left\{v_{1}, v_{2}, \ldots, v_{p}\right\}, V \subseteq Y$. Содержательно будем считать, что задача $I=<X, V, \rho>$ состоит в перечислении для произвольно взятого запроса $x \in X$ всех тех и только тех записей $y$ из $V$, которые содержат $x$ в качестве подслова, то есть для которых выполнено $x \rho y$. Назовём эту задачу задачей поиска подслова. Множество $V$ в дальнейшем будем называть библиотекой. Так как $X$ и $\rho$ фиксированы, задача поиска подслова полностью определяется библиотекой. Похожая задача рассматривалась в [3].

Введём понятие информационного графа (ИГ) в соответствии с $[1,2]$. В формальном определении понятия ИГ используются два описанных выше множества $X$ и $Y$, а также множество $F$ предикатов, заданных на множестве $X$ (предикаты - это функции, которые могут принимать только два значения: 0 или 1), и множество $G$ переключателей, заданных на множестве $X$ (переключатели - это функции, область значений которых является начальным отрезком натурального ряда). Пару $\mathcal{F}=\langle F, G>$ будем называть базовым множеством. Рассмотрим произвольный ориентированный граф. Выделим в нём одну вершину. Назовём её корнем. Выделим в графе какие-либо другие вершины. Назовём их листьями. Сопоставим каждому листу некоторую запись из множества $Y$. Это соответствие назовем нагрузкой листьев. Выделим в графе некоторые вершины (это могут быть в том числе корень и листья) и назовем их точками переключения. Если $\beta$ - вершина графа, то через $\psi_{\beta}$ обозначим полустепень исхода вершины $\beta$ (то есть число исходящих из этой вершины рёбер).Каждой точке переключения $\beta$ сопоставим некий символ из $G$. Это соответствие назовем нагрузкой точек переключения. Для каждой точки переключения $\beta$ ребрам, из нее исходящим, поставим во взаимно однозначное соответствие числа из множества $\left\{1,2, \ldots, \psi_{\beta}\right\}$. Эти ребра назовем переключательны- 
ми, а это соответствие - нагрузкой переключательных ребер. Ребра, не являющиеся переключательными, назовем предикатными. Каждому предикатному ребру графа сопоставим некоторый символ из множества $F$. Это соответствие назовем нагрузкой предикатных ребер. Полученную нагруженную сеть назовем информационным графом над базовым множеством $\mathcal{F}=\langle F, G\rangle$.

Определим функционирование ИГ. Скажем, что предикатное ребро проводит запрос $x \in X$, если предикат, приписанный этому ребру, принимает значение 1 на запросе $x$; переключательное ребро, которому приписан номер $r$, проводит запрос $x \in X$, если переключатель, приписанный началу этого ребра, принимает значение $r$ на запросе $x$; ориентированная цепь ребер проводит запрос $x \in X$, если каждое ребро цепи проводит запрос $x$; запрос $x \in X$ проходит в вершину $\beta$ ИГ, если существует ориентированная цепь, ведущая из корня в вершину $\beta$, которая проводит запрос $x$; запись $y$, приписанная листу $\alpha$, попадает (включается) в ответ ИГ на запрос $x \in X$, если запрос $x$ проходит в лист $\alpha$. Ответом ИГ $u$ на запрос $x$ назовем множество записей, попавших в ответ ИГ на запрос $x$, и обозначим его $J_{u}(x)$. Эту функцию $J_{u}(x)$ будем считать результатом функционирования ИГ $u$ и называть функцией ответа ИГ $u$. Тем самым понятие ИГ полностью определено.

Назовём каждую цепь, по которой запрос $x$ проходит из корня в какой-либо лист, существенной цепью ИГ, соответствующей запросу $x$.

Скажем, что ИГ $u$ решает ЗИП $I=\{X, V, \rho\}$, если для любого запроса $x \in X$ ответ на этот запрос содержит все те и только те записи из $V$, которые удовлетворяют запросу $x$, то есть $J_{u}(x)=\{y \in V: x \rho y\}$.

Определим понятие сложности информационного графа на запросе. Пусть $N(f, u, x)$ - количество вычислений функции $f$ при обработке в ИГ $u$ запроса $x$, a $t(f, x)$ - отображение, ставящее каждой паре $(f, x)$, где $f$ - функция, $x$ - запрос, в соответствие некоторое положительное число (будем считать, что это число характеризует время вычисления функции $f$ на запросе $x$ ). Сложностью вычисления ИГ $u$ на запросе $x$ назовём число $T(u, x)=\sum_{f \in F \cup G} N(f, u, x) t(f, x) . T(u, x)$ характеризует время обработки запроса $x$.

Объемом $Q(u)$ ИГ $u$ назовем число ребер в ИГ $u$. Величина $Q(u)$ соответствует объёму памяти, используемой информационным графом.

В качестве базового множества $\mathcal{F}$ будем рассматривать $<F, G>, F=\{f(x)\}$, $G=\left\{g_{i}(x), i \in \mathbb{N}\right\}$, где $f(x) \equiv 1, g_{i}(x)=x[i]$, если $x[i] \neq 0$, и $g_{i}(x)=k+1$, если $x[i]=0$. С помощью предиката, тождественно равного 1 , моделируется перечисление ответа на запрос. Вычисление этого предиката соответствует переходу по ссылке (например, к слову из ответа), в то время как вычисление любого переключателя $g_{i}(x)$ включает в себя выделение $i$-й буквы слова $x$ и переход по ссылке. По этой причине сложность вычисления предиката, тождественно равного 1 , считается положительной, но меньшей, чем сложность вычисления переключателя $g_{i}(x)$. Будем считать, что сложность вычисления любого переключателя $g_{i}(x)$ равна 1 , a сложность вычисления предиката $f(x)$ равна $t, 0<t<1$.

Введём следующие обозначения. $U(I, \mathcal{F})$ - множество всех ИГ над базовым множеством $\mathcal{F}$, решающих ЗИП $I=<X, V, \rho>$; $d(I, x)=|\{v \in V, x \rho v\}|$, где $I=<X, V, \rho>; e(x)=\min (l(x)+1, n) ; R(I, \mathcal{F}, x)=e(x)+t(d(I, x)-1)$.

Теорема 1. При $k \geqslant 3$ среди всех библиотек $V \subseteq N_{k}^{n}$ из $p$ слов, где $p \leqslant(k-1)^{n}$,

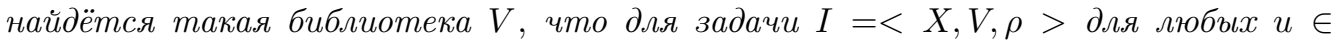


$U(I, \mathcal{F})$ и $x \in X$ выполняется следующее условие: если $d(I, x) \geqslant 1, \operatorname{mo~} T(u, x) \geqslant$ $R(I, \mathcal{F}, x)$.

Обозначим через $U_{I}$ множество таких графов $u \in U(I, \mathcal{F})$, что для каждого из них при любых $x$ из $X$ выполняется условие $T(u, x) \leqslant R(I, \mathcal{F}, x)$. Как следует из теоремы 1 , при $k \geqslant 3, p \leqslant(k-1)^{n}$ для некоторой библиотеки $V$ из $p$ слов любой ИГ из множества $U_{I}$ является самым быстрым для любого запроса $x$, которому удовлетворяет хотя бы одно слово из библиотеки.

Теорема 2. Для любой задачи $I=<X, V, \rho>$ множество $U_{I}$ не пусто.

Скажем, что функция $f(p, n): \mathbb{N} \times \mathbb{N} \rightarrow \mathbb{R}_{>0}$ асимптотически не превосходит функцию $g(p, n): \mathbb{N} \times \mathbb{N} \rightarrow \mathbb{R}_{>0}$ при $p \rightarrow \infty, n \rightarrow \infty$ и обозначим $f(p, n) \lesssim g(p, n)$, если существует такие функция $\alpha(p, n): \mathbb{N} \times \mathbb{N} \rightarrow \mathbb{R}, \alpha(p, n) \rightarrow 0$ при $n \rightarrow \infty, p \rightarrow \infty$ и числа $p_{0} \in \mathbb{N}, n_{0} \in \mathbb{N}$, что $f(p, n) \leqslant(1+\alpha(p, n)) * g(p, n)$, когда $p>p_{0}, n>n_{0}$.

Пусть $Q(p, n)=\max _{I: I=\langle X, V, \rho>,|V|=p} \min _{u \in U_{I}} Q(u)$.

Теорема 3. Eсли $k \geqslant 3, p \leqslant(k-1)^{[n / 3]}$, mo $p n^{2} / 9 \lesssim Q(p, n) \lesssim(k+2) p n^{2} / 2 n p u$ $n \rightarrow \infty, p \rightarrow \infty$.

\section{3. Доказательство теоремы 1}

Лемма 1. Пусть $k \geqslant 3$ и в библиотеке $V \subseteq N_{k}^{n}$ из $p$ слов, где $p \leqslant(k-1)^{n}$, каждая буква каждого слова отлична от $k$. Тогда для любого ИГ $u \in U(I, \mathcal{F})$, где $I=<X, V, \rho>, u$ для каждого запроса $x \in X$, которому удовлетворяет хотя бъ одно слово из библиотеки, выполнено неравенство $T(u, x) \geqslant R(I, \mathcal{F}, x)$.

Доказательство. Рассмотрим произвольную библиотеку $V$, удовлетворяющую условиям леммы, и произвольный ИГ $u \in U(I, \mathcal{F})$, где $I=<X, V, \rho>$. Покажем сначала, что если $d(I, x) \geqslant 1$, то $T(u, x) \geqslant l(x)$. Рассмотрим какую-либо цепь $C$, по которой запрос проходит из корня в лист с записью $v$, удовлетворяющей запросу (назовём этот лист $\gamma$ ), причём ни одна вершина этой цепи, кроме $\gamma$, не является листом. Пусть существует такое $i \in \mathbb{N}, 1 \leqslant i \leqslant l(x)$, что ни одной из вершин $C$, исключая $\gamma$, не сопоставлен переключатель $g_{i}(x)$. Тогда заменим $x[i]$ на $k$. Получившийся запрос также будет проходить в лист с записью $v$. Мы имеем противоречие с тем, что ни в одном слове из $V$ нет буквы $k$, а следовательно, и любого подслова, содержащего букву $k$. Получим, что для любого $i \in \mathbb{N}, 1 \leqslant i \leqslant l(x)$ найдётся вершина цепи, не совпадающая с $\gamma$ и такая, что ей сопоставлен $g_{i}$. Значит, $T(u, x) \geqslant l(x)$.

Далее, пусть $l(x)<n$. Тогда по крайней мере одной из вершин $C$, не считая $\gamma$, должен быть сопоставлен переключатель $g_{l(x)+1}$. Если это не так, заменим $x[l(x)+1]$ на $k$ и снова придём к противоречию. Следовательно, $T(u, x) \geqslant l(x)+1$.

Пусть $d(I, x)>1$. Это возможно только тогда, когда $l(x)<n$. Для каждого отличного от $\gamma$ листа, в который проходит запрос $x$, выберем ребро, по которому $x$ входит в этот лист. Так как никакому листу не может быть сопоставлено более одной записи, все эти рёбра разные, причём если некоторые из этих рёбер исходят из одной вершины, то это предикатные рёбра. Кроме того, ни одно из этих рёбер не принадлежит $C$. Значит, для прохода запроса по таким рёбрам будет вычислено не менее $d(I, x)-1$ функций. Кроме того, при обработке запроса будет вычислено не менее $l(x)+1$ указанных ранее переключателей. Значит, $T(u, x) \geqslant l(x)+1+t$. $(d(I, x)-1)$. Итак, мы показали, что если $d(I, x) \geqslant 1$, то $T(u, x) \geqslant R(I, \mathcal{F}, x)$. 
Теорема 1 непосредственно вытекает из леммы 1.

\section{4. Доказательство теоремы 2}

Покажем, что для любой библиотеки $V$ можно построить такой ИГ $u_{1}(I, \mathcal{F})$, peшающий задачу $I=<X, V, \rho>$ над базовым множеством $\mathcal{F}$, что для всех $x \in X$ выполняется $T\left(u_{1}, x\right) \leqslant R(I, \mathcal{F}, x)$. Далее часто вместо $u_{1}(I, \mathcal{F})$ будем писать $u_{1}$, предполагая, что $I$ и $\mathcal{F}$ зафиксированы.

Пусть $V=\left\{v_{1}, \ldots, v_{p}\right\}$. Через $V^{\prime}$ обозначим множество всех подслов всех слов из $V$. Будем строить информационный граф $u_{1}$ индукцией по длине $s$ слов из $V^{\prime}$.

Базис индукции: $s=1$. Выберем произвольную вершину, объявим её корнем информационного графа. Выберем ещё $k+1$ вершин, и в каждую из них направим из корня ребро. Пронумеруем эти рёбра числами от 1 до $k+1$. Сопоставим корню переключатель $g_{1}(x)$. Рассмотрим все слова длины 1 из $V^{\prime}$. Пусть это слова $v_{1}^{1}, v_{2}^{1}, \ldots, v_{p_{1}}^{1}$. Заметим, что так как $v_{i}^{1} \neq v_{j}^{1}, i \neq j$, то $v_{i}^{1}[1] \neq v_{j}^{1}[1], i \neq j$. Для каждого $i \in\left\{1,2, \ldots, p_{1}\right\}$ проделаем следующее. Найдём вершину, в которую из корня ведёт ребро под номером $v_{i}^{1}[1]$, назовём её $\beta_{v_{i}^{1}}$. Выберем $k+1$ новых вершин и в каждую из них направим из $\beta_{v_{i}^{1}}$ ребро. Пронумеруем эти рёбра числами от 1 до $k+1$. Вершину, в которую ведёт $(k+1)$-е исходящее из $\beta_{v_{i}^{1}}$ ребро, назовём $\gamma_{v_{i}^{1}}$. Сопоставим вершине $\beta_{v_{i}^{1}}$ переключатель $g_{2}(x)$. Рассмотрим теперь все слова из $V$, в которых $v_{i}^{1}$ содержится в качестве подслова (хотя бы одно такое слово найдётся по построению $V^{\prime}$ ). Пусть это слова $v_{j_{1, i, 1}}, \ldots, v_{j_{1, i, p}, i}$, где $j_{1, i, a}<j_{1, i, b}$, когда $a<b$. Слово $v_{j_{1, i, 1}}$ сопоставим вершине $\gamma_{v_{i}^{1}}$. Каждое из оставшихся слов из $V$, содержащих $v_{i}^{1}$ в качестве подслова, сопоставим новой вершине. Для каждого из слов $v_{j_{1, i, a}}, a \in\left\{1, \ldots, p_{1, i}-1\right\}$, выпустим из вершины, которой это слово приписано, в вершину, которой приписано слово $v_{j_{1, i, a+1}}$, ребро с предикатом $f(x)$.

Шаг индукции: пусть $2 \leqslant s \leqslant n$, и мы уже рассмотрели все слова из $V^{\prime}$, имеющие длину $1,2, \ldots, s-1$, причём для каждого такого слова $v^{\prime}$ в графе найдётся вершина $\beta_{v^{\prime}}$. Рассмотрим все слова длины $s$ из $V^{\prime}$. Пусть это слова $v_{1}^{s}, v_{2}^{s}, \ldots, v_{p_{s}}^{s}$. Для каждого $i \in\left\{1,2, \ldots, p_{s}\right\}$ проделаем следующее. Для каждого $v_{i}^{s}$ его подслово $v_{i}^{s}[1 . . s-1]$, очевидно, принадлежит $V^{\prime}$, значит, по предположению индукции в ИГ есть вершина $\beta_{v_{i}^{s}[1 . . s-1]}$. Заметим, что так как $v_{i}^{s} \neq v_{j}^{s}$, когда $i \neq j$, то либо $v_{i}^{s}[1 . . s-1] \neq v_{j}^{s}[1 . . s-1]$, либо $v_{i}^{s}[s] \neq v_{j}^{s}[s]$, когда $i \neq j$. Возможны 2 случая:

1) $s<n$. Рассмотрим вершину, в которую из $\beta_{v_{i}^{s}[1 . . s-1]}$ ведёт ребро под номером $v_{i}^{s}[s]$. Назовём её $\beta_{v_{i}^{s}}$. Выберем $k+1$ новых вершин, и в каждую из них направим из $\beta_{v_{i}^{s}}$ ребро. Пронумеруем эти рёбра числами от 1 до $k+1$. Вершину, в которую ведёт $(k+1)$-е исходящее из $\beta_{v_{i}^{s}}$ ребро, назовём $\gamma_{v_{i}^{s}}$. Сопоставим вершине $\beta_{v_{i}^{s}}$ переключатель $g_{s+1}(x)$.

2) $s=n$. Рассмотрим вершину, в которую из $\beta_{v_{i}^{s}[1 . . s-1]}$ ведёт ребро под номером $v_{i}^{s}[s]$. Назовём её $\gamma_{v_{i}^{s}}$.

Рассмотрим теперь все слова из $V$, в которых $v_{i}^{s}$ содержится в качестве подслова (хотя бы одно такое слово найдётся по построению $V^{\prime}$ ). Пусть это слова $v_{j_{s, i, 1}}, \ldots, v_{j_{s, i, p_{s, i}}}$ где $j_{s, i, a}<j_{s, i, b}$, когда $a<b$. Слово $v_{j_{s, i, 1}}$ сопоставим вершине $\gamma_{v_{i}^{s}}$. Каждое из оставшихся слов из $V$, содержащих $v_{i}^{s}$ в качестве подслова, сопоставим новой вершине. Для каждого из слов $v_{j_{s, i, a}}$, где $a \in\left\{1, \ldots, p_{s, i}-1\right\}$, выпустим из вершины, которой это слово приписано, в вершину, которой приписано слово $v_{j_{s, i, a+1}}$, ребро с предикатом $f(x)$. Шаг индукции на этом закончен.

Тем самым построение ИГ $u_{1}$ завершено. 
Лемма 2. Информационный граф $u_{1}(I, \mathcal{F})$ решает задачу $I=<X, V, \rho>$ над базовым множеством $\mathcal{F}$.

Доказательство. Заметим сначала, что так как из каждой предикатной вершины в графе выходит не более одного ребра, то из каждой вершины, куда прошёл запрос, он может перейти не более чем по одному ребру. Кроме того, граф является деревом (поскольку при построении графа новые рёбра всякий раз направлялись в новые вершины).

Будем доказывать лемму индукцией по длине запроса.

Базис индукции. Рассмотрим какой-либо запрос $x$ длины 1 . Пусть найдутся такие $i \in\{1, \ldots, p\}$ и $j \in\{1, \ldots, n\}$, что $x=v_{i}[j . . j]$. Тогда $x \in V^{\prime}$, то есть существует $q \in\left\{1, \ldots, p_{1}\right\}$ такое, что $x=v_{q}^{1}$ и в графе найдётся вершина $\beta_{x}$, притом запрос $x$ проходит из корня в эту вершину после вычисления переключателя $g_{1}(x)$. Затем запрос проходит в вершину $\gamma_{x}\left(g_{2}(x)=k+1\right.$, так как $\left.x[2]=0\right)$. Этой вершине сопоставлено одно из слов, удовлетворяющих запросу $x$. Далее на каждом шаге запрос переходит по предикатному ребру с предикатом $f(x)$, тождественно равном единице, в вершину, которой приписано следующее слово, удовлетворяющее запросу, пока не попадёт в вершину со словом $v_{j_{1, q, p}, q}$. Из этой вершины не выходит ни одно ребро. Таким образом, в ответ на запрос попадут все записи, удовлетворяющие $x$, и никакие другие. Пусть теперь для всех $i \in\{1, \ldots, p\}$ и $j \in\{1, \ldots, n\}$ выполнено условие $x \neq v_{i}[j . . j]$. Тогда в графе нет вершины $\beta_{x}$, и вершине, в которую запрос пройдёт из корня, не приписано ни одно слово, притом из этой вершины не выходит ни одно ребро. Значит, ни одна запись не попадёт в ответ на запрос $x$. Итак, действительно, информационный граф решает задачу для запросов длины 1.

Предположим, что ИГ решает задачу для всех запросов длины не более $s-1$, где $s \in\{2, \ldots, n\}$, причём: а) каждый такой запрос $w$ пройдёт в вершину $\beta_{w}$ тогда и только тогда, когда в библиотеке найдётся хотя бы одно слово, удовлетворяющее этому запросу; б) если запрос $w$ проходит в вершину $\beta_{w}$, то в вершинах цепи, по которой он туда проходит, не считая самой $\beta_{w}$, последовательно вычисляются переключатели $g_{1}, \ldots, g_{l(w)}$, и только они. Мы уже показали, что для слов длины 1 условия а) и б) выполнены. Рассмотрим произвольное слово $x$ длины $s$. Пусть существуют такие $i \in\{1, \ldots, p\}$ и $j \in\{1, \ldots, n\}$, что $x=v_{i}[j . . j+s-1]$. Тогда $x \in V^{\prime}$ и найдётся такое $q$, что $x=v_{q}^{s}$. Для слова $x^{\prime}=x[1 . . s-1]$ в графе найдётся вершина $\beta_{x^{\prime}}$. По предположению индукции в вершинах (за исключением $\beta_{x^{\prime}}$ ) пути, по которому запрос $x^{\prime}$ проходит в $\beta_{x^{\prime}}$, последовательно вычисляются переключатели $g_{1}, \ldots, g_{s-1}$, и только они. Значит, запрос $x$ также проходит в вершину $\beta_{x^{\prime}}$. В этой вершине вычисляется переключатель $g_{s}$. Если $s=n$, то запрос $x$ проходит в вершину $\gamma_{x}$; если же $s<n$, то $x$ проходит в вершину $\beta_{x}$, а уже из неё, после вычисления переключателя $g_{s+1}$, который на запросе $x$ примет значение $k+1$, запрос пройдёт в $\gamma_{x}$. Вершине $\gamma_{x}$ сопоставлено одно из слов, удовлетворяющих запросу $x$. Далее на каждом шаге запрос переходит по предикатному ребру с предикатом $f$, тождественно равном единице, в вершину, которой приписано следующее слово, удовлетворяющее запросу, пока не попадёт в вершину со словом $v_{j_{s, q, p}, q}$. Из этой вершины не выходит ни одно ребро. Таким образом, в ответ на запрос попадут все записи, удовлетворяющие $x$, и никакие другие. Пусть теперь для всех $i \in\{1, \ldots, p\}$ и $j \in\{1, \ldots, n\}$ выполнено условие $x \neq v_{i}[j . . j+s-1]$. Возможны 2 случая:

1) Для всех $v^{\prime} \in V^{\prime}$ выполнено $x[1 . .1] \neq v^{\prime}$. Тогда вершине, в которую запрос пройдёт из корня, не приписано ни одно слово, притом из этой вершины не выходит ни одно ребро. Значит, ни одна запись не попадёт в ответ на запрос $x$. 
2) Выполнено условие $x[1 . .1] \in V^{\prime}$. Рассмотрим $l=\max _{x\left[1 . . l^{\prime}\right] \in V^{\prime}} l^{\prime}$. Очевидно, $1 \leqslant l \leqslant s-1$. Тогда в графе есть вершина $\beta_{x[1 . . l]}$, причём запрос $x$ проходит из корня в эту вершину. Из $\beta_{x[1 . . l]}$ запрос пройдёт в вершину, которой не приписано ни одно слово, притом такую, что из неё не выходит ни одно ребро. Значит, ни одна запись не попадёт в ответ на запрос $x$.

Итак, действительно, построенный информационный граф $u_{1}$ решает задачу $I$.

Лемма 3. Для любого запроса $x \in X$ выполнено $T\left(u_{1}(I, \mathcal{F}), x\right) \leqslant R(I, \mathcal{F}, x)$.

Доказательство. Оценим сложность $u_{1}$ на произвольном запросе $x$. Возможны следующие случаи.

1) Ни одно слово из $V$ не содержит $x$ в качестве подслова. Тогда найдётся такое $s \in\{1, \ldots, l(x)\}$, что при обработке запроса будут последовательно вычислены переключатели $g_{1}, g_{2}, \ldots, g_{s}$, причём в результате вычисления $g_{s}$ запрос пройдёт в вершину, которой не приписано ни одно слово и из которой не выходит ни одно ребро. Значит, сложность обработки такого запроса $x$ не превысит $l(x)$.

2) $l(x)=n$ и в библиотеке $V$ есть слово, содержащее $x$ в качестве подслова (значит, $x \in V^{\prime}$, так как длина каждой записи из библиотеки равна $n$ ). Тогда при обработке запроса будут последовательно вычислены переключатели $g_{1}, g_{2}, \ldots, g_{n}$, причём в результате вычисления $g_{n}$ запрос пройдёт в вершину $\gamma_{x}$, которой сопоставлено слово $x$. Из этой вершины не выходит ни одно ребро, так как других слов, кроме $x$, удовлетворяющих запросу, в библиотеке быть не может. Следовательно, сложность обработки такого запроса $x$ равна $l(x)$.

3) $l(x)<n$ и в библиотеке $V$ есть слово, содержащее $x$ в качестве подслова. Тогда при обработке запроса будут последовательно вычислены переключатели $g_{1}, g_{2}, \ldots, g_{l(x)+1}$, причём в результате вычисления $g_{l(x)+1}$ запрос пройдёт в вершину $\gamma_{x}$, которой сопоставлено какое-либо слово из $V$, удовлетворяющее $x$. Далее запрос последовательно (по рёбрам с предикатами $f(x)$ ) проходит в вершины, которым сопоставлены все остальные слова, удовлетворяющие запросу. Значит, сложность обработки такого запроса $x$ равна $l(x)+1+t \cdot(d(I, x)-1)$.

Итак, мы показали, что $u_{1} \in U(I, \mathcal{F})$ и для любого $x$ выполнено $T\left(u_{1}, x\right) \leqslant$ $R(I, \mathcal{F}, x)$. Следовательно, $u_{1} \in U_{I}$, то есть $U_{I} \neq \varnothing$.

Перейдём к непосредственному доказательству теоремы 2. Согласно лемме 2, при любой библиотеке $V \subseteq Y$ ИГ $u_{1}(I, \mathcal{F})$, где $I=<X, V, \rho>$, решает задачу $I$ над базовым множеством $\mathcal{F}$. Согласно лемме 3 , для любого запроса $x \in X$ выполнено $T\left(u_{1}(I, \mathcal{F}), x\right) \leqslant R(I, \mathcal{F}, x)$. Значит, $u_{1} \in U_{I}$. Теорема 2 доказана.

\section{5. Доказательство теоремы 3}

Лемма 4. Если $I=<X, V, \rho>,|V|=p, \operatorname{mo} Q\left(u_{1}(I, \mathcal{F})\right) \leqslant(k+2) p(n+2)(n-1) / 2+$ $p+k+1$.

Доказательство. ИГ $u_{1}$ содержит 3 группы ребер.

1) Каждому слову $v^{\prime} \in V^{\prime}$ сопоставлена вершина $\gamma_{v^{\prime}}$, причём в эту вершину проходит только запрос $v^{\prime}$. Значит, каждому слову $v^{\prime}$ можно поставить в соответствие переключательное ребро, ведущее в $\gamma_{v^{\prime}}$, а также каждое ребро, по которому запрос $v^{\prime}$ пройдёт после попадания в $\gamma_{v^{\prime}}$. Значит, каждому $v^{\prime}$ будет сопоставлено столько рёбер, сколько слов из $V$ содержат $v^{\prime}$ в качестве подслова. Суммарно для всех слов 
из $V^{\prime}$ таких рёбер будет не более общего числа подслов всех слов из библиотеки $V$, то есть не более $p \cdot(1+2+\ldots+n)=p n(n+1) / 2$.

2 ) Кроме того, каждому слову $v^{\prime}$, имеющему длину не более $n-1$, сопоставлена вершина $\beta_{v^{\prime}}$. Следовательно, каждому такому $v^{\prime}$ можно поставить в соответствие все рёбра, выходящие из $\beta_{v^{\prime}}$, за исключением рёбер, ведущих в какие-либо вершины $\gamma_{v^{\prime \prime}}$, то есть не более $k+1$ рёбер. Так как в $V^{\prime}$ не более $p n(n+1) / 2$ слов, мы получим, что рёбер, рассматриваемых в данном пункте, в графе не больше $(k+1) p(n(n+1) / 2-$ $1)=(k+1) p(n+2)(n-1) / 2$.

3) Кроме рёбер, рассмотренных в пунктах 1) и 2), в графе есть ещё только рёбра, выходящие из корня. Их $k+1$.

Итак, мы получили, что $Q\left(u_{1}\right) \leqslant(k+2) p(n+2)(n-1) / 2+p+k+1$.

Построим такую библиотеку $V$, что если $u \in U_{I}$, где $I=<X, V, \rho>$, то $Q(u) \gtrsim$ $p n^{2} / 9, n \rightarrow \infty, p \rightarrow \infty$.

Пусть $k \geqslant 3, n \geqslant 3$. Обозначим $r=[n / 3]$.

Рассмотрим все слова $w_{1}, \ldots, w_{(k-1)^{r}}$ длины $r$, не содержащие букву $k$, причём $w_{i}<w_{j}$, если $i<j$. Определим каждое слово $v_{i}, i \in N_{k-1}^{r}$, следующим образом:

$$
\begin{cases}v_{i}[r+1 . .2 r]=w_{i} & \\ v_{i}[r]=v_{i}[2 r+1]=1 & \text { если } j \in \mathbb{N}, j \leqslant r-1 \\ v_{i}[r-j]=(j-1) \bmod (k-2)+2, & \text { если } j \in \mathbb{N}, j \leqslant n-2 r-1 \\ v_{i}[2 r+j+1]=(j-1) \bmod (k-2)+2, & \end{cases}
$$

Пример: пусть $n=14, k=5$. Тогда $r=4, w_{5}=1121, v_{5}=43211121123423$.

Рассмотрим слова $v_{i, a, b}=v_{i}[a . . b]$, где $a \in \mathbb{N}, b \in \mathbb{N}, a \leqslant r, 2 r+1 \leqslant b \leqslant n$, $i \in\left\{1, \ldots,(k-1)^{r}\right\}$.

Лемма 5. $v_{i_{1}, a_{1}, b_{1}}=v_{i_{2}, a_{2}, b_{2}}$ тогда и только тогда, когда $i_{1}=i_{2}, a_{1}=a_{2}, b_{1}=b_{2}$.

Доказательство. Достаточность, очевидно, следует из определения слов $v_{i, a, b}$. Докажем необходимость. Пусть $v_{i_{1}, a_{1}, b_{1}}=v_{i_{2}, a_{2}, b_{2}}$. По определению $v_{i, a, b}$ имеем: $\quad v_{i_{1}, a_{1}, b_{1}}\left[r-a_{1}+1\right]=v_{i}[r]=1, v_{i_{1}, a_{1}, b_{1}}[j] \neq 1, j \leqslant r-a_{1}$. Аналогично $v_{i_{2}, a_{2}, b_{2}}\left[r-a_{2}+1\right]=1, v_{i_{2}, a_{2}, b_{2}}[j] \neq 1, j \leqslant r-a_{2}$. Значит, $r-a_{1}+1=r-a_{2}+1$, откуда $a_{1}=a_{2}$. Так как $b_{1}-a_{1}+1=l\left(v_{i_{1}, a_{1}, b_{1}}\right)=l\left(v_{i_{2}, a_{2}, b_{2}}\right)=b_{2}-a_{2}+1$, то $b_{1}=b_{2}$. Далее, имеем $v_{i_{1}, a_{1}, b_{1}}\left[r-a_{1}+2 . .2 r-a_{1}+1\right]=v_{i_{1}}[r+1 . .2 r]=w_{i_{1}}$, $v_{i_{2}, a_{2}, b_{2}}\left[r-a_{1}+2 . .2 r-a_{1}+1\right]=v_{i_{2}, a_{2}, b_{2}}\left[r-a_{2}+2 . .2 r-a_{2}+1\right]=v_{i_{2}}[r+1 . .2 r]=w_{i_{2}}$, то есть $w_{i_{1}}=w_{i_{2}}$, откуда $i_{1}=i_{2}$. Необходимость доказана.

Итак, любые 2 слова вида $v_{i, a, b}$, у которых не совпадает хотя бы одно из значений $i, a, b$, разные.

Лемма 6. Пусть $k \geqslant 3$ и в библиотеке $V \subseteq N_{k}^{n}$ из р слов, где $p \leqslant(k-1)^{n}$, каждая буква каждого слова отлична от $k$, пусть также $u \in U(I, \mathcal{F})$, где $I=<X, V, \rho>$, и для каждого запроса $x \in X$, которому удовлетворяет хотя би одно слово из библиотеки, выполнено $T(u, x)=R(I, \mathcal{F}, x)$. Тогда:

1) каждой из первых е(x) вершин (считая от корня) любой существенной цепи информационного графа, соответствующей $x$, сопоставлен один из переключателей $g_{i}$, где $i \in\{1, \ldots, e(x)\}$, причём каждый переключатель $g_{i}$ сопоставлен ровно одной вершине;

2) первые $е(x)+1$ вериин (считая от корня) всех существенных иепей информационного графа, соответствующих $x$, общие;

3 Дискретная математика, т. 26 № 2 
3) $($ (е(x)+1)-я и все последующие вершины (считая от корня) любой существенной цепи информачионного графа, соответствующей $x$, являются листьями;

4) каждое выходящее из листа, в который проходит запрос $x$, ребро любой существенной цепи информационного графа, соответствующей $x$, является предикатным.

Доказательство. Предположим, что для каждого запроса $x$, для которого в библиотеке найдётся хотя бы одно удовлетворяющее ему слово, выполнено $T(u, x)=$ $R(I, \mathcal{F}, x)$. Как показано в доказательстве леммы 1, при обработке запроса хотя бы по одному разу должен быть вычислен каждый из переключателей $g_{1}(x), \ldots, g_{e(x)}(x)$. Кроме того, для попадания запроса в каждый лист должно быть вычислено ещё не менее $d(I, x)-1$ функций. Значит, если $T(u, x)=R(I, \mathcal{F}, x)$, то каждый из переключателей $g_{1}(x), \ldots, g_{e(x)}(x)$ при обработке запроса должен быть вычислен ровно по одному разу, а остальных функций должно быть вычислено ровно $d(I, x)-1$. Так как $t<1$, эти функции являются предикатами. Соответствующие этим предикатам рёбра должны входить в листья. Следовательно, каждый предикат при обработке запроса вычисляется уже после того, как вычислены все переключатели $g_{1}(x), \ldots, g_{e(x)}(x)$. Значит, в любой существенной цепи рассматриваемого ИГ, соответствующей $x$, все эти переключатели сопоставлены первым $e(x)$ вершинам (считая от корня). Таким образом, п.1 обоснован.

Далее, заметим, что из каждой из первых $e(x)$ вершин, в которые проходит запрос $x$, данный запрос переходит ровно по одному ребру, так как все эти вершины - переключательные. Значит, первые $e(x)+1$ вершин (считая от корня) всех существенных цепей информационного графа, соответствующих $x$, общие. П.2 обоснован.

Так как слов, удовлетворяющих запросу $x$, всего $d(I, x)$, а предикатных рёбер в графе $d(I, x)-1$, то найдётся лист, в который запрос $x$ проходит по переключательному ребру. Значит, этот лист является $(e(x)+1)$-й вершиной (считая от корня) каждой существенной цепи информационного графа, соответствующей $x$. Далее, в рассматриваемом графе всего $e(x)$ переключательных вершин, притом в каждой существенной цепи информационного графа, соответствующей $x$, это вершины с номерами $1,2, \ldots, e(x)$, считая от корня. Значит, все рёбра, выходящие из остальных вершин каждой такой цепи, являются предикатными. В рассматриваемом ИГ каждое предикатное ребро входит в лист, следовательно, в каждой существенной цепи информационного графа, соответствующей $x$, вершины с номерами, большими $e(x)$ (считая от корня), являются листьями. П.3 обоснован.

Как уже показано, каждый лист, в который проходит запрос $x$, должен иметь номер не меньше $e(x)+1$, считая от корня, в какой-либо существенной цепи ИГ, соответствующей запросу $x$. Значит, каждое выходящее из этого листа ребро является предикатным, то есть п.4 также обоснован.

Лемма 7. Пусть $k \geqslant 3$ и в библиотеке $V \subseteq N_{k}^{n}$ из $p$ слов, где $p \leqslant(k-1)^{n}$, каждая буква каждого слова отлична от $k$, пусть также $u \in U(I, \mathcal{F})$, где $I=\langle X, V, \rho>, u$ для каждого запроса $x \in X$, которому удовлетворяет хотя бы одно слово из библиотеки, выполнено $T(u, x)=R(I, \mathcal{F}, x)$. Тогда в каждой существенной цепи информационного графа, соответствующей запросу $x$, переключатель $g_{i}, i \in\{2, \ldots, e(x)\}$, сопоставлен вершине с номером $i-1$ или $i$ (считая от корня), а переключатель $g_{1}$ может быть сопоставлен вершине с любым номером om 1 до e $(x)$. 
Доказательство. Заметим, что согласно пунктам 1 и 2 леммы 6 , у каждой существенной цепи информационного графа, соответствующей запросу $x$, первые $e(x)$ вершин (считая от корня) общие, причём этим вершинам сопоставлены переключатели $g_{i}, i \in\{1, \ldots, e(x)\}$. Обозначим часть любой из этих существенных цепей от корня до $e(x)$-й вершины через $D=D(x)$. Докажем лемму индукцией по $l(x)$.

Базис индукции. Пусть $l(x)=1$. Тогда в $D$ две вершины: 1 -я и 2-я. Им должны быть приписаны переключатели $g_{1}$ и $g_{2}$, притом они могут идти в любом порядке. Как видно, условие леммы для базиса индукции выполнено.

Шаг индукции. Пусть теперь условие леммы выполняется для всех запросов длины меньше $s$. Докажем, что оно выполнено для всех запросов длины $s$. Рассмотрим запрос $x, l(x)=s$. Обозначим $x^{\prime}=x[1 . . l(x)-1]$. Рассмотрим цепь $D^{\prime}=D\left(x^{\prime}\right)$. Заметим, что результат вычисления всех переключателей, кроме $g_{l(x)}$, на запросах $x$ и $x^{\prime}$ одинаков, а результат вычисления $g_{l(x)}$ на этих запросах разный (соответственно $x[l(x)]$ и $k+1)$. Значит, часть цепи $D^{\prime}$ от корня до вершины $\beta$ с переключателем $g_{l(x)}$ является частью цепи $D$. Вершина $\beta$ по предположению индукции имеет в цепи $D^{\prime}$ номер $l(x)-1$ либо $l(x)$. Все вершины части цепи $D^{\prime}$ от корня до $\beta$ сохраняют свои номера и в цепи $D$. Рассмотрим оба случая.

1) Вершина $\beta$ имеет номер $l(x)-1$. Тогда в части цепи $D$ от корня до $\beta$ имеются вершины с переключателями $g_{2}, \ldots, g_{l(x)}$, но нет вершин с переключателями $g_{1}$ и $g_{l(x)+1}$. Если $l(x)<n$, то у $D l(x)+1$ вершина, и вершинам с номерами $l(x)$ и $l(x)+1$ должны быть приписаны переключатели $g_{1}$ и $g_{l(x)+1}$, притом они могут идти в любом порядке. Если $l(x)=n$, то у $D l(x)$ вершин, и вершине с номером $l(x)$ должен быть сопоставлен переключатель $g_{1}$.

2 ) Вершина $\beta$ имеет номер $l(x)$. Тогда $D^{\prime}-$ часть $D$. В части цепи $D$ от корня до $\beta$ имеются вершины с переключателями $g_{1}, \ldots, g_{l(x)}$. Если $l(x)=n$, то у $D l(x)$ вершин, поэтому $D$ и $D^{\prime}$ совпадают. Если же $l(x)<n$, то у $D$ имеется $l(x)+1$ вершин, и вершине с номером $l(x)+1$ должен быть приписан переключатель $g_{l(x)+1}$.

Этим исчерпываются все возможные случаи.

Перейдём к непосредственному доказательству теоремы 3.

Везде далее будем рассматривать библиотеку $V=\left\{v_{i}, i \in\{1, \ldots, p\}\right\}$, каждая запись $v_{i}$ которой определена согласно соотношению (1), и произвольный граф $u \in$ $U_{I}$, где $I=<X, V, \rho>$. Заметим, что библиотека $V$ удовлетворяет условиям лемм 1,6 и 7.

Рассмотрим цепь $C_{1}$, по которой какой-либо запрос $x_{1}$ с $d\left(I, x_{1}\right)>0$ проходит из корня в лист (назовём этот лист $\gamma_{1}$ ) и которая не содержит никакого другого листа. Рассмотрим также цепь $C_{2}$, по которой какой-либо другой запрос $x_{2} \mathrm{c} d\left(I, x_{2}\right)>0$ проходит из корня в лист (назовём этот лист $\gamma_{2}$ ) и которая не содержит никакого другого листа. Пусть в графе найдутся 2 цепи $A_{1}$ и $A_{2}$, по которым $x_{1}$ и $x_{2}$ проходят из корня в одну и ту же переключательную вершину $\alpha$, причём рёбра, по которым эти запросы проходят непосредственно в $\alpha$, не совпадают. Пусть в $A_{1}$ имеется $l_{1}$ вершин, считая $\alpha$, в $A_{2}$ имеется $l_{2}$ вершин, считая $\alpha$. Не ограничивая общности, считаем, что $l_{1} \leqslant l_{2}$. Заметим, что $l_{2} \geqslant l_{1}>1$. По лемме 7 имеем: вершине $\alpha$ сопоставлен, с одной стороны, один из переключателей $g_{1}, g_{l_{1}}, g_{l_{1}+1}$, а с другой стороны - переключатель из множества $\left\{g_{1}, g_{l_{2}}, g_{l_{2}+1}\right\}$. Возможны следующие случаи.

1) Вершине $\alpha$ сопоставлен переключатель $g_{l_{1}+1}$, совпадающий с переключателем $g_{l_{2}}$. То есть $l_{2}=l_{1}+1$. Из леммы 7 вытекает, что для каждого $i \in\left\{1, \ldots, l_{2}-1\right\}$ среди первых (считая от корня) $l_{2}-1$ вершин цепи $A_{2}$ найдётся вершина с переключателем 
$g_{i}$. Рассмотрим запрос $x_{3}=x_{2}\left[1 . . l_{2}-1\right]$. Так как $d\left(x_{2}\right)>0$, то $d\left(x_{3}\right)>0$. Запрос $x_{3}$ пройдёт в вершину $\alpha$, а из неё по ребру $k+1-$ в лист $\beta$, которому приписано слово $v$, удовлетворяющее запросу $x_{3}$. Далее, из леммы 7 вытекает, что для любого $i \in$ $\left\{2, \ldots, l_{1}\right\}$ среди первых $l_{1}-1$ вершин цепи $A_{1}$ найдётся вершина с переключателем $g_{i}$, а вершин с переключателем $g_{1}$ нет. Рассмотрим запрос $x_{4}$, полученный из $x_{1}\left[1 . . l_{1}\right]$ заменой первой буквы на $k$. Тогда этот запрос пройдёт по цепи $A_{1}$ в вершину $\alpha$, а из неё по ребру $k+1-$ в $\beta$. Следовательно, $x_{4}$ пройдёт в лист, которому приписано слово $v$, чего быть не должно, так как в $v$ нет буквы $k$. Значит, случай 1) невозможен.

2) Вершине $\alpha$ сопоставлен переключатель $g_{l_{1}}$, совпадающий с переключателем $g_{l_{2}}$. То есть $l_{2}=l_{1}$. Как и в предыдущем случае, запрос $x_{4}=x_{2}\left[1 . . l_{2}-1\right]$ пройдёт в вершину $\alpha$. В эту же вершину пройдёт слово $x_{3}=x_{1}\left[1 . . l_{1}-1\right]$. Заметим, что $x_{4} \neq x_{3}$, так как иначе эти запросы попали бы в $\alpha$ по одной и той же цепи. Рассмотрим все слова $v \in V$. Положим $q_{1, v}$ равным $\min \left\{q: v\left[q . . q+l_{1}-2\right]=x_{3}\right\}$, если подслово $x_{3}$ содержится в $v$, и $n+1$ в противном случае. Аналогично положим $q_{2, v}=\min \{q$ : $\left.v\left[q . . q+l_{1}-2\right]=x_{4}\right\}$, если подслово $x_{4}$ содержится в $v$, и $n+1$ в противном случае. Пусть $q^{\prime}=\min _{v \in V} \min \left(q_{1, v}, q_{2, v}\right)$. Тогда $q^{\prime}<n+1$, так как и для запроса $x_{3}$, и для $x_{4}$ найдётся в $V$ хотя бы одно слово, удовлетворяющее этому запросу. Не ограничивая общности, будем считать, что слово $w \in V$ таково, что $w\left[q^{\prime} . . q^{\prime}+l_{1}-2\right]=x_{3}$ и цепь $C$, по которой запрос $x_{5}=w\left[q^{\prime} . . n\right]$ проходит из корня в лист $\gamma$ со словом $w$, не содержит других листьев с записями, удовлетворяющими запросу $x_{5}$. Тогда цепь $A_{1}$ является началом цепи $C$. Следовательно, на отрезке цепи $C$ от $\alpha$ до $\gamma$ есть вершины с переключателями $g_{i}, i \in\left\{l_{1}, \ldots, \min \left(n-q^{\prime}+2, n\right)\right\}$, и нет вершин с другими переключателями. Рассмотрим запрос $x_{6}$, полученный из $x_{5}$ заменой первых $l_{1}-1$ букв на соответствующие буквы слова $x_{4}$. Запрос $x_{6}$ пройдёт по цепи $A_{2}$ в вершину $\alpha$, а затем по отрезку цепи $C$ от $\alpha$ до $\gamma-$ в вершину $\gamma$. Таким образом, $x_{6}$ является подсловом слова $w$. Так как $l\left(x_{6}\right)=n-q^{\prime}+1$ и $x_{6} \neq x_{5}$, то должно найтись такое число $q^{\prime \prime}<q^{\prime}$, что $w\left[q^{\prime \prime} . . q^{\prime \prime}-q^{\prime}+n\right]=x_{6}$. Но, учитывая, что $x_{3}-$ начало $x_{6}$, это противоречит выбору $q^{\prime}$. Значит, случай 2) невозможен.

3) Вершине $\alpha$ сопоставлен переключатель $g_{l_{1}+1}$, совпадающий с переключателем $g_{l_{2}+1}$. То есть $l_{2}=l_{1}<n$. Из леммы 7 вытекает, что для любого $i \in\left\{2, \ldots, l_{1}\right\}$ среди первых $l_{1}-1$ вершин цепи $A_{1}$ найдётся вершина с переключателем $g_{i}$, а вершин с переключателем $g_{1}$ нет. Это же верно и для $A_{2}$. Запрос $x_{3}=x_{1}\left[1 . . l_{1}\right]$ пройдёт в вершину $\alpha$, а из неё по ребру $k+1-$ в какую-либо вершину $\beta$, причём $\beta$ должен быть сопоставлен переключатель $g_{1}$. Рассмотрим запрос $x_{4}$, полученный из $x_{2}\left[1 . . l_{1}\right]$ заменой первой буквы на $x_{1}[1]$. Дальнейшие рассуждения повторяют рассуждения из предыдущего пункта с небольшими изменениями. Заметим, что $x_{3} \neq x_{4}$, так как иначе эти запросы должны были бы пройти в $\alpha$ по одному и тому же пути, чего не происходит. Для любого слова $v \in V$ положим $q_{1, v}$ равным $\min \left\{q: v\left[q . . q+l_{1}-2\right]=\right.$ $\left.x_{3}\right\}$, если подслово $x_{3}$ содержится в $v$, и $n+1$ в противном случае. Аналогично положим $q_{2, v}=\min \left\{q: v\left[q . . q+l_{1}-2\right]=x_{4}\right\}$, если подслово $x_{4}$ содержится в $v$, и $n+1$ в противном случае. Пусть $q^{\prime}=\min _{v \in V} \min \left(q_{1, v}, q_{2, v}\right)$. Тогда $q^{\prime}<n+1$, так как для запроса $x_{3}$ найдётся в $V$ хотя бы одно слово, удовлетворяющее этому запросу. Не ограничивая общности, будем считать, что слово $w \in V$ таково, что $w\left[q^{\prime} . . q^{\prime}+l_{1}-1\right]=$ $x_{3}$ и цепь $C$, по которой запрос $x_{5}=w\left[q^{\prime} . . n\right]$ проходит из корня в лист $\gamma$ со словом $w$, не содержит других листьев с записями, удовлетворяющими запросу $x_{5}$. Тогда цепь $A_{1}$ является началом цепи $C$. Следовательно, на отрезке цепи $C$ от $\alpha$ до $\gamma$ есть вершины с переключателями $g_{i}, i \in\left\{l_{1}+1, \ldots, \min \left(n-q^{\prime}+2, n\right)\right\}$, а также вершина с 
переключателем $g_{1}$, и нет вершин с другими переключателями. Рассмотрим запрос $x_{6}$, полученный из $x_{5}$ заменой первых $l_{1}$ букв на соответствующие буквы слова $x_{4}$. Запрос $x_{6}$ пройдёт по цепи $A_{2}$ в вершину $\alpha$, а затем по отрезку цепи $C$ от $\alpha$ до $\gamma-$ в вершину $\gamma$. Таким образом, $x_{6}$ является подсловом слова $w$. Так как $l\left(x_{6}\right)=n-q^{\prime}+1$ и $x_{6} \neq x_{5}$, то должно найтись такое число $q^{\prime \prime}<q^{\prime}$, что $w\left[q^{\prime \prime} . . q^{\prime \prime}-q^{\prime}+n\right]=x_{6}$. Но, поскольку $x_{4}$ - начало $x_{6}$, это противоречит выбору $q^{\prime}$. Значит, случай 3 ) невозможен.

4) Вершине $\alpha$ сопоставлен переключатель $g_{1}$. Согласно лемме 7 , для любого $i \in\left\{2, \ldots, l_{1}\right\}$ среди первых $l_{1}-1$ вершин цепи $A_{1}$ найдётся вершина с переключателем $g_{i}$ и для любого $i \in\left\{2, \ldots, l_{2}\right\}$ среди первых $l_{2}-1$ вершин цепи $A_{2}$ найдётся вершина с переключателем $g_{i}$. Рассмотрим цепь $C_{i}$, по которой запрос $x_{i}, i \in\{1,2\}$, проходит из корня в какой-либо лист $\gamma_{i}$ и которая не содержит других листьев. При этом цепи $C_{1}$ и $C_{2}$ содержат соответственно цепи $A_{1}$ и $A_{2}$ и поэтому не совпадают. Скажем, что вершина обладает $O$-свойством относительно цепи $C_{i}$, если ей сопоставлен переключатель $g_{1}$ и она является ближайшей к $\gamma_{i}$ переключательной вершиной цепи $C_{i}$. Случай 4$)$ можно разбить на несколько подслучаев.

$4.1)$ Вершина $\alpha$ не обладает $O$-свойством ни относительно цепи $C_{1}$, ни относительно цепи $C_{2}$. Пусть запрос $x_{3}=x_{1}\left[1 . . l_{1}\right]$ проходит из $\alpha$ в переключательную вершину $\beta$. Из леммы 7 вытекает, что этой вершине может быть сопоставлен только переключатель $g_{l_{2}+1}$. Рассмотрим запрос $x_{4}$, полученный из запроса $x_{2}\left[1 . . l_{2}\right]$ заменой первой буквы на $x_{1}[1]$. Этот запрос пройдёт из корня в $\alpha$ по цепи $A_{2}$, а далее в $\beta$ по ребру $x_{1}[1]$. Поэтому вершине $\beta$ должен быть сопоставлен переключатель $g_{l_{1}+1}$, откуда получаем, что $l_{1}=l_{2}$. Далее практически без изменений повторяются рассуждения, приведённые в п.3. Заметим, что $x_{3} \neq x_{4}$, так как иначе эти запросы должны были бы пройти в $\alpha$ по одному и тому же пути, чего не происходит. Для любого слова $v \in V$ положим $q_{1, v}$ равным $\min \left\{q: v\left[q . . q+l_{1}-1\right]=x_{3}\right\}$, если подслово $x_{3}$ содержится в $v$, и $n+1$ в противном случае. Аналогично положим $q_{2, v}=\min \left\{q: v\left[q . . q+l_{1}-1\right]=x_{4}\right\}$, если подслово $x_{4}$ содержится в $v$, и $n+1$ в противном случае. Пусть $q^{\prime}=\min _{v \in V} \min \left(q_{1, v}, q_{2, v}\right)$. Тогда $q^{\prime}<n+1$, так как для запроса $x_{3}$ найдётся в $V$ хотя бы одно слово, удовлетворяющее этому запросу. Не ограничивая общности, будем считать, что слово $w \in V$ таково, что $w\left[q^{\prime} . . q^{\prime}+l_{1}-1\right]=x_{3}$ и цепь $C$, по которой запрос $x_{5}=w\left[q^{\prime} . . n\right]$ проходит из корня в лист $\gamma$ со словом $w$, не содержит других листьев с записями, удовлетворяющими запросу $x_{5}$. Тогда цепь $A_{1}$ является началом цепи $C$. Следовательно, на отрезке цепи $C$ от $\beta$ до $\gamma$ есть вершины с переключателями $g_{i}, i \in\left\{l_{1}+1, \ldots, \min \left(n-q^{\prime}+2, n\right)\right\}$, и нет вершин с другими переключателями. Рассмотрим запрос $x_{6}$, полученный из $x_{5}$ заменой первых $l_{1}$ букв на соответствующие буквы слова $x_{4}$. Запрос $x_{6}$ пройдёт по цепи $A_{2}$ в вершину $\alpha$, потом - в $\beta$ по ребру $x_{2}[1]$, а затем по отрезку цепи $C$ от $\beta$ до $\gamma-$ в вершину $\gamma$. Таким образом, $x_{6}$ является подсловом слова $w$. Так как $l\left(x_{6}\right)=n-q^{\prime}+1$ и $x_{6} \neq x_{5}$, то должно найтись такое число $q^{\prime \prime}<q^{\prime}$, что $w\left[q^{\prime \prime} . . q^{\prime \prime}-q^{\prime}+n\right]=x_{6}$. Но, поскольку $x_{4}$ - начало $x_{6}$, это противоречит выбору $q^{\prime}$. Значит, случай 4.1) невозможен.

$4.2)$ Вершина $\alpha$ обладает $O$-свойством относительно цепи $C_{i}$, но не относительно $C_{j}$, где $i, j \in\{1,2\}, i \neq j$. Тогда, в частности, $l_{j}<n$. Запрос $x_{i}$ проходит из $\alpha$ непосредственно в $\gamma_{i}$. Рассмотрим такой запрос $x_{3}$, что $x_{3}[1]=x_{i}[1], x_{3}\left[2 . . l_{j}\right]=$ $x_{j}\left[2 . l_{j}\right], x_{3}\left[l_{j}+1\right]=k$. Этот запрос пройдёт по цепи $A_{j}$ в вершину $\alpha$, оттуда по ребру $x_{i}[1]-$ в вершину $\gamma_{i}$. Значит, слово, сопоставленное $\gamma_{i}$, должно удовлетворять этому запросу, чего быть не может, так как оно не содержит буквы $x_{3}\left[l_{j}+1\right]=k$. 
$4.3)$ Вершина $\alpha$ обладает $O$-свойством и относительно цепи $C_{1}$, и относительно $C_{2}$. Такую вершину будем называть вершиной слияния.

Итак, из всех возможных вариантов мы не исключили только 4.3.

Ранее мы показали, что все слова $v_{i, a, b}=v_{i}[a . . b], 1 \leqslant a \leqslant r, 2 r+1 \leqslant b \leqslant n, 1 \leqslant i \leqslant p$, разные. Значит, все слова $v_{i, a, b}$, у которых $a<r$, также разные; обозначим через $V^{\prime}$ множество таких слов. Как нетрудно проверить, $\left|V^{\prime}\right|=(r-1) \cdot(n-2 r) \cdot p$. Для каждого запроса $x_{i}$ из $V^{\prime}, i \in\left\{1, \ldots,\left|V^{\prime}\right|\right\}$, рассмотрим цепь $C_{i}$, по которой запрос $x_{i}$ проходит из корня в какой-либо лист $\gamma_{i}$ со словом, удовлетворяющим запросу, и которая не содержит других листьев с записями, удовлетворяющими этому запросу. Выделим для каждого $x_{i}$ такое ребро, что никакой запрос $x_{j} \in V^{\prime}$ не проходит по этому ребру, если $j \neq i$.

Если $C_{i}$ не содержит вершин слияния, то в качестве такого ребра можно выбрать последнее (считая от корня) переключательное ребро $c_{i}$ цепи $C_{i}$. Действительно, пусть это ребро выходит из вершины $\alpha$. Тогда для любого $i \in\left\{1, \ldots, \min \left(l\left(x_{i}+1\right), n\right)\right\}$ найдётся вершина цепи $C_{i}$ от корня до $\alpha$ с переключателем $g_{i}$, причём в каждую вершину этого отрезка цепи $C_{i}$, кроме корня, входит одно ребро (в корень - 0 рёбер). Значит, для того, чтобы произвольный запрос $x$ прошёл по ребру $c_{i}$, должны выполняться равенства $x[1]=x_{i}[1], \ldots, x\left[l\left(x_{i}\right)\right]=x_{i}\left[l\left(x_{i}\right)\right]$, а также, если $l\left(x_{i}\right)<n$, равенство $x\left[l\left(x_{i}\right)+1\right]=x_{i}\left[l\left(x_{i}\right)+1\right]=0$. Следовательно, $x=x_{i}$.

Пусть $C_{i}$ содержит вершину слияния. Очевидно, ни в какой цепи не может быть более одной такой вершины. Назовём эту вершину $\alpha$. Тогда в качестве нужного ребра можно выбрать ребро $e_{i}$ цепи $C_{i}$, входящее в $\alpha$. Действительно, для того, чтобы произвольный запрос $x$ прошёл по ребру $e_{i}$, должны выполняться равенства $x[2]=x_{i}[2], \ldots, x\left[l\left(x_{i}\right)\right]=x_{i}\left[l\left(x_{i}\right)\right]$, а также, если $l\left(x_{i}\right)<n$, равенство $x\left[l\left(x_{i}\right)+1\right]=$ $x_{i}\left[l\left(x_{i}\right)+1\right]=0$. Но слова из $V^{\prime}$ подобраны так, что из равенства вторых букв любых двух слов из $V^{\prime}$ следует равенство их первых букв. Значит, снова получаем $x=x_{i}$.

Итак, для каждого слова из $V^{\prime}$ мы выделили в ИГ $u$ ребро, соответствующее этому слову и не соответствующее никаким другим словам из $V^{\prime}$. Значит, рёбер в $u$ не меньше $\left|V^{\prime}\right|=(r-1) \cdot(n-2 r) \cdot p$. Таким образом, $p n^{2} / 9 \lesssim Q(p, n), n \rightarrow \infty, p \rightarrow \infty$.

Согласно лемме 2 при любой библиотеке $V \subseteq Y$ ИГ $u_{1}(I, \mathcal{F})$, где $I=<X, V, \rho>$, решает задачу $I$ над базовым множеством $\mathcal{F}$. Согласно лемме 3 для любого запроса $x$ выполнено неравенство $T\left(u_{1}(I, \mathcal{F}), x\right) \leqslant R(I, \mathcal{F}, x)$. Значит, $u_{1} \in U_{I}$. Тогда $Q\left(u_{1}\right) \leqslant$ $(k+2) p(n+2)(n-1) / 2+p+k+1$ согласно лемме 4. Следовательно, $Q(p, n) \lesssim$ $(k+2) p n^{2} / 2, n \rightarrow \infty, p \rightarrow \infty$.

Имеем $p n^{2} / 9 \lesssim Q(p, n) \lesssim(k+2) p n^{2} / 2, n \rightarrow \infty, p \rightarrow \infty$, что и требовалось.

Автор выражает благодарность профессору Эльяру Эльдаровичу Гасанову за научное руководство и помощь в научной работе.

\section{Список литературы}

1. Гасанов Э.Э., Кудрявцев В.Б., Теория хранения и поиска информации, Физматлит, Москва, 2002.

2. Кудрявцев В.Б., Гасанов Э.Э., Подколзин А.С., Введение в теорию интеллектуальных систем, Издательский отдел факультета ВМиК МГУ, Москва, 2006, 94-117.

3. Перпер Е.М., "О функциональной сложности поиска подстроки", Интеллектуалъные системы, 15:1-4 (2011), 553-570. 